\title{
Detección de la hipertensión arterial pulmonar en esclerosis sistémica basada en la evidencia: Estudio DETECT
}

\section{Evidence-based detection of pulmonary arterial hypertension in systemic sclerosis: DETECT Trial}

Pablo Ramón GIL ToRres, MD. ${ }^{(1)}$; Claudio VillaquiRÁn TORRES, MD. ${ }^{(2)}$

(1)Residente de tercer año de Medicina Interna, Universidad ICESI, Fundación Valle del Lili, Cali, Colombia.

(2)Internista, Neumólogo, Hospital Universitario San Ignacio, Pontificia Universidad Javeriana, Bogotá, Colombia.

Correspondencia: Claudio Villaquirán Torres, correo electrónico: claudiovillaquiran@ gmail.com

Recibido: 15/08/14. Aceptado: 10/09/14.

\begin{abstract}
Referencia
Coghlan JG, Denton CP, Grünig E, Bonderman D, Distler O, Khanna D. The DETECT study group. Evidencebased detection of pulmonary arterial hypertension in systemic sclerosis. Ann Rheum Dis. 2014;73:1340-9.
\end{abstract}

\section{Pregunta}

En pacientes con esclerosis sistémica y alto riesgo de desarrollar hipertensión arterial pulmonar, ¿es posible desarrollar un algoritmo de detección basado en evidencia que tenga mejor rendimiento diagnóstico que las recomendaciones de la guía de la Sociedad Europea de Cardiología/Sociedad Europea de Medicina Respiratoria (ESC/ERS) para la detección temprana de hipertensión arterial pulmonar?

\section{Diseño}

Estudio de corte transversal, multicéntrico. DETECT (de la sigla del inglés Detection of Pulmonary Arterial Hypertensin in Systemic Sclerosis).

\section{Cegamiento}

No aplica.

\section{Periodo de alistamiento}

Entre 2008 y 2011.

\section{Lugar}

62 centros especializados de 18 ciudades en Estados Unidos, Europa y Asia.

\section{Pacientes}

Se incluyeron pacientes mayores de 18 años con diagnóstico de esclerosis sistémica (acorde con los criterios del Colegio Americano de Reumatología, incluyendo pacientes con otras enfermedades del tejido conectivo que cumplieran esos criterios) con alto riesgo de desarrollar hipertensión arterial pulmonar (más de tres años de evolución a partir del primer síntoma no Raynaud y capacidad de difusión de monóxido de carbono -DLCOpredicha $<60 \%$ ).

Se excluyeron pacientes con hipertensión pulmonar confirmada mediante cateterismo cardíaco derecho previo al inicio del estudio, que recibieran tratamiento reconocido para hipertensión pulmonar y/o que tuvieran una capacidad vital forzada $(\mathrm{CVF})<40 \%$ del 
predicho, falla renal, evidencia previa de enfermedad cardíaca izquierda clínicamente relevante o estado de embarazo.

\section{Intervención}

Se evaluaron 112 variables potencialmente asociadas con hipertensión arterial pulmonar en esclerosis sistémica, agrupadas en cuatro categorías: parámetros clínicos y demográficos y exámenes de laboratorio, electrocardiografía y ecocardiografía. Se practicó cateterismo cardíaco derecho a todos los pacientes luego de la recolección de la información previamente descrita. Se realizó un análisis estadístico en tres etapas: con el modelo de regresión logística uni y multivariable se seleccionaron variables candidatas asociadas a hipertensión arterial pulmonar; posteriormente estas fueron reducidas empleando la regresión logística multivariable y el concepto del comité científico sobre su confiabilidad y plausibilidad clínica. Finalmente se construyó un algoritmo de decisión en dos pasos con base en dos modelos de regresión logística multivariable. Se calculó el área bajo la curva ROC para evaluar la capacidad discriminativa del algoritmo de decisión respecto a la diferenciación de pacientes con hipertensión arterial pulmonar y sin hipertensión pulmonar. Además, se evaluó un algoritmo alternativo con especificidad del $65 \%$ en su segundo paso y se comparó el desempeño con relación a las recomendaciones de las guías ESC/ERS en la población del estudio.

\section{Resultados}

Se tamizaron 646 pacientes con esclerosis sistémica de los cuales 158 no lograron los criterios de elegibilidad (en su mayoría por tener DLCO > 60\%). De 488 pacientes restantes, 466 fueron llevados a cateterismo cardiaco derecho el cual reveló hipertensión pulmonar en 145 (31\%) e hipertensión arterial pulmonar en 87 (19\%). Los resultados se enfocaron en esos 87 pacientes con hipertensión arterial pulmonar vs. los 321 pacientes sin hipertensión pulmonar.
Se construyó un algoritmo de decisión en dos pasos: el primero incluyó seis variables no ecocardiográficas para generar un resultado de predicción que permitiera la exclusión de pacientes con bajo riesgo de tener hipertensión arterial pulmonar (sensibilidad asignada 97\%) y determinara la orden de toma de ecocardiografía. Estas variables fueron: $\mathrm{CVF} \%$ predicho/DLCO\% predicho, tener o haber tenido telangiectasias, presencia de anticuerpos anticentrómero, valor del NT pro BNP sérico, urato sérico y desviación del eje a la derecha en el electrocardiograma.

En el segundo paso (especificidad asignada del $35 \%$ ) la puntuación del primer paso fue combinada con dos variables ecocardiográficas para generar un puntaje de predicción de riesgo de tener hipertensión arterial pulmonar y así determinar si el paciente debía ser referido a cateterismo cardiaco derecho para el diagnóstico. Estas variables fueron: área de la aurícula derecha y velocidad del jet de regurgitación tricuspídea.

Se practicó el algoritmo a los 408 pacientes (87 con hipertensión arterial pulmonar y 321 sin hipertensión pulmonar) tomados en cuenta para el estudio con los resultados observados en la figura 1 .

La capacidad discriminatoria combinada para el paso uno (puntuación >300) expresada como el área bajo la curva ROC fue de $84,4 \%$ (95\% IC 79,5 $89,8 \%$ ). El área bajo la curva ROC para el total de la puntuación de riesgo del paso uno y las dos variables ecocardiográficas seleccionadas (paso dos si puntuación > 35) fue de $88,1 \%$ (IC 95\% 82,4-92,3\%). De los 408 pacientes inicialmente seleccionados, 304 pasaron al segundo paso y 52 no. De estos últimos, 2 fueron falsos negativos (tenían hipertensión arterial pulmonar en el cateterismo cardíaco derecho). Posteriormente, de los 304 pacientes que pasaron al segundo paso, 198 cumplieron los criterios de remisión a cateterismo cardíaco derecho y 69 no. De esos 69, un paciente fue falso negativo. De los 198 pacientes restantes, 69 tuvieron hipertensión arterial pulmonar en el cateterismo cardíaco derecho. En general, el algoritmo tuvo tres falsos negativos (4\%) y 69 verdaderos positivos. 


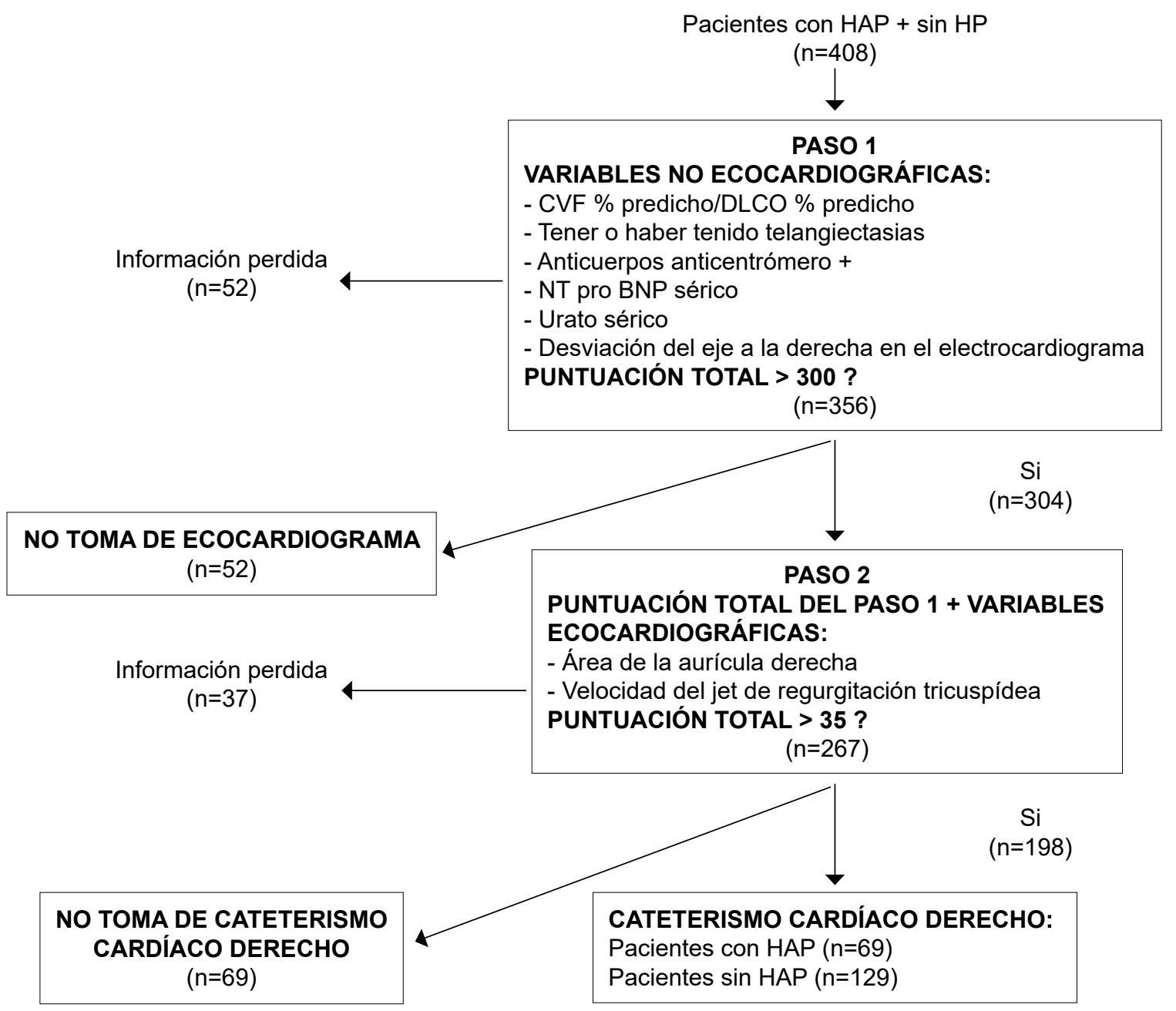

Figura 1.

La exclusión de una sola variable del algoritmo DETECT tuvo un impacto mínimo en su desempeño. No obstante, si se pierde más de una variable el modelo no puede ser usado de forma confiable. En la práctica clínica, en caso de perder la información de una variable a esta se le debe asignar una puntuación de 50 (excepto para tener o haber tenido telangiectasias a la que se asignan 65 puntos).

Comparado con las guías ESC/ERS para detección de hipertensión arterial pulmonar, el algoritmo DETECT tuvo menos falsos negativos (4\% vs. $29 \%$ ). La proporción de cateterismo cardíaco derecho que no confirmó el diagnóstico de hipertesnión arterial pulmonar (falsos positivos) fue similar entre el algoritmo DETECT y las guías ESC/ERS (65 vs. 60\%). Al aplicar el algoritmo DETECT se recomendó que al $62 \%$ de los pacientes se le practicara cateterismo cardíaco derecho, una mayor proporción que si se emplean las recomendaciones de la guía ESC/ERS (40\%). No obstante, cuando se empleó la modificación del paso 2 (especificidad del 65\%) con indicación de cateterismo cardíaco derecho solo si la puntuación es mayor a 40, el algoritmo recomendó ordenarlo a un $41 \%$ de los pacientes a costa de un incremento de los falsos negativos de 4 al 15\%, lo cual es aún más bajo que los falsos negativos cuando se usa la guía ESC/ ERS (29\%). 
Tabla 1. Comparación del modelo DETECT y las guías ECS/ERS.

\begin{tabular}{|lcccccc|}
\hline & $\begin{array}{c}\text { Tasa de } \\
\text { remisión a } \\
\text { CCD }\end{array}$ & $\begin{array}{c}\text { Tasa de } \\
\text { falsos } \\
\text { negativos }\end{array}$ & $\begin{array}{c}\text { Sensibilidad } \\
\text { (\%) }\end{array}$ & $\begin{array}{c}\text { Especificidad } \\
\text { (\%) }\end{array}$ & $\begin{array}{c}\text { Valor } \\
\text { predictivo } \\
\text { positivo (\%) }\end{array}$ & $\begin{array}{c}\text { Valor } \\
\text { predictivo } \\
\text { negativo (\%) }\end{array}$ \\
$\begin{array}{l}\text { Análisis primario del } \\
\text { algoritmo DETECT } \\
\text { (n=319) }\end{array}$ & 62 & 4 & 96 & 48 & 35 & 98 \\
$\begin{array}{l}\text { Análisis del } \\
\text { algoritmo DETECT } \\
\text { con especificidad } \\
\text { del 65\% en el paso }\end{array}$ & 41 & 15 & 85 & 72 & 47 & 94 \\
$2(n=319)$ & & & & & & \\
$\begin{array}{l}\text { Guías ECS/ERS } \\
(n=371)^{*}\end{array}$ & 40 & 29 & 71 & 69 & 40 & 89 \\
\hline
\end{tabular}

*Evaluado en su subgrupo de pacientes con información disponible para las variables definidas en la guía con los siguientes criterios para remisión a cateterismo cardíaco derecho, CCD: velocidad de regurgitación del jet tricuspídeo mayor a 3,4 m/s o velocidad entre 2,8 y 3,4 $\mathrm{m} / \mathrm{s}$ y síntomas (dolor anginoso, síncope/casi síncope, disnea, edema periférico) o velocidad $<2,8 \mathrm{~m} / \mathrm{s}$ y síntomas (descritos atrás) y la presencia de variables ecocardiográficas adicionales sugerentes de hipertensión pulmonar (área de la aurícula derecha mayor a $16 \mathrm{~cm}^{2} \mathrm{y} / \mathrm{o}$ radio de diámetro ventricular derecho/diámetro de fin de diástole del ventrículo izquierdo mayor a 0,8 ).

\section{Conclusión}

El nuevo modelo basado en la evidencia para la detección no invasiva de la hipertensión arterial pulmonar en pacientes con esclerosis sistémica, es una herramienta sensible, con baja tasa de falsos negativos, que permite la identificación temprana de la enfermedad y direcciona su tratamiento.

\section{Comentario}

Las iniciativas de tamización previas $(1,2) \sin$ la realización sistemática de cateterismo cardiaco derecho dieron lugar a las recomendaciones de las guías (3) basadas en síntomas y resultados del ecocardiograma sin la información del número de diagnósticos de hipertensión arterial pulmonar perdidos (falsos negativos). Este es el primer estudio sobre detección de hipertensión arterial pulmonar que hizo cateterismo cardiaco derecho de forma sistemática a todos los pacientes y desarrolló un algoritmo basado en evidencia empleando información clínica que está a la mano y una prueba no invasiva (ecocardiograma) validada (4) para identificar de forma temprana la hipertensión arterial pulmonar en una población con síntomas mínimos.

El uso de este algoritmo diagnóstico en la práctica clínica es factible dada la simplicidad de las variables que emplea y su uso en dos pasos. Esto permite minimizar el número de diagnósticos de hipertensión arterial pulmonar perdidos. No obstante, se debe tener en cuenta que el grupo seleccionado al cual se debe aplicar es a los pacientes con esclerosis sistémica y alto riesgo de desarrollar hipertensión arterial pulmonar, es decir, a aquellos con más de tres años de evolución a partir del primer síntoma no Raynaud y capacidad DLCO predicha menor al $60 \%$.

El algoritmo diagnóstico del estudio DETECT cuenta con alta sensibilidad (96\%) aunque con baja especificidad (48\%), lo que llevó a un mayor número de indicaciones de cateterismo cardíaco derecho en la población estudiada cuando se comparó con las recomendaciones de la ESC/ ERS. No obstante, la proporción de cateterismo cardíaco derecho que no confirmó un diagnóstico de hipertensión arterial pulmonar fue similar entre ambas estrategias (65 vs. 60\%). Además, la proporción de indicación de cateterismo cardíaco derecho mediante el algoritmo DETECT respecto a las guías ESC/ERS, puede ser menor debido a que estas últimas se aplican a la población general con esclerosis sistémica sin la especificación para la población en alto riesgo de desarrollar hipertensión arterial pulmonar. Cuando se ajustó la 
especificidad de este algoritmo para recomendar cateterismo cardíaco derecho en una proporción similar a las guías ESC/ERS, se mantuvo un número menor de diagnósticos de hipertensión arterial pulmonar perdidos (falsos negativos).

Finalmente, vale aclarar que este estudio es de corte transversal así que no es posible determinar el desempeño del algoritmo a largo tiempo o dar lugar a recomendaciones sobre qué tan frecuentemente deben ser evaluados los pacientes. De igual forma, al ser seleccionados solo pacientes con esclerosis sistémica, su aplicación a la población general puede dar lugar a una alta tasa de falsos negativos para los demás grupos de hipertensión pulmonar de la Organización Mundial de la Salud. De esta forma, su aplicabilidad se basa en pacientes con esclerosis sistémica y alto riesgo de hipertensión arterial pulmonar optimizando el empleo de los recursos en el diagnóstico de esta población y permitiendo su intervención temprana con un mejor pronóstico $(5,6)$. La calculadora de riesgo con este algoritmo está disponible de forma gratuita en Google Play y App Store bajo el título "detect PAH".

\section{Conflictos de intereses}

Los autores declaran no tener conflictos de interés.

\section{Bibliografía}

1. Hachulla E, Gressin V, Guillevin L, Carpentier P, Diot E, Sibilia J. Early detection of pulmonary arterial hypertension in systemic sclerosis: a French nationwide prospective multicenter study. Arthritis Rheum. 2005;52:3792-800.

2. Mukerjee D, St George D, Coleiro B, Knight C, Denton CP, Davar J. Prevalence and outcome in systemic sclerosis associated pulmonary arterial hypertension: application of a registry approach. Ann Rheum Dis. 2003;62:1088-93.

3. Galiè N, Hoeper MM, Humbert M, Torbicki A, Vachiery JL, Barbera JA. Guidelines for the diagnosis and treatment of pulmonary hypertension: the Task Force for the Diagnosis and Treatment of Pulmonary Hypertension of the European Society of Cardiology (ESC) and the European Respiratory Society (ERS), endorsed by the International Society of Heart and Lung Transplantation (ISHLT). Eur Heart J. 2009;30:2493-537.

4. Mukerjee D, St George D, Knight C, Davar J, Wells AU, Du Bois RM, et al. Echocardiography and pulmonary function as screening tests for pulmonary arterial hypertension in systemic sclerosis. Rheumatology. 2004;43:461-6.

5. Humbert M, Yaici A, de Groote P, Montani D, Sitbon O, Launay D. Screening for pulmonary arterial hypertension in patients with systemic sclerosis: clinical characteristics at diagnosis and long-term survival. Arthritis Rheum. 2011;63:3522-30.

6. Galiè N, Rubin Lj, Hoeper M, Jansa P, Al-Hiti H, Meyer G. Treatment of patients with mildly symptomatic pulmonary arterial hypertension with bosentan (EARLY study): a double-blind randomized controlled trial. Lancet. 2008;371:2093-100. 